\title{
Exome sequencing improves genetic diagnosis of structural fetal abnormalities revealed by ultrasound
}

\author{
Keren J. Carss ${ }^{1}$, Sarah C. Hillman², Vijaya Parthiban¹, Dominic J. McMullan ${ }^{3}$, \\ Eamonn R. Maher², Mark D. Kilby2,4,* and Matthew E. Hurles ${ }^{1}$ \\ ${ }^{1}$ Wellcome Trust Sanger Institute, Wellcome Trust Genome Campus, Hinxton, Cambridgeshire CB10 1SA, UK, ${ }^{2}$ School \\ of Clinical and Experimental Medicine (Birmingham Centre for Women's and Children's Health), College of Medical \\ and Dental Sciences, University of Birmingham, Edgbaston, Birmingham B15 2TT, UK, ${ }^{3}$ West Midlands Regional \\ Genetics Laboratory, Birmingham Women's NHS Trust, Edgbaston, Birmingham B15 2TG, UK and ${ }^{4}$ Fetal Medicine \\ Centre, Birmingham Women's Foundation Trust, Edgbaston, Birmingham B15 2TG, UK
}

Received November 14, 2013; Revised January 10, 2014; Accepted January 23, 2014

The genetic etiology of non-aneuploid fetal structural abnormalities is typically investigated by karyotyping and array-based detection of microscopically detectable rearrangements, and submicroscopic copy-number variants (CNVs), which collectively yield a pathogenic finding in up to $10 \%$ of cases. We propose that exome sequencing may substantially increase the identification of underlying etiologies. We performed exome sequencing on a cohort of 30 non-aneuploid fetuses and neonates (along with their parents) with diverse structural abnormalities first identified by prenatal ultrasound. We identified candidate pathogenic variants with a range of inheritance models, and evaluated these in the context of detailed phenotypic information. We identified 35 de novo single-nucleotide variants (SNVs), small indels, deletions or duplications, of which three (accounting for $10 \%$ of the cohort) are highly likely to be causative. These are de novo missense variants in FGFR3 and COL2A1, and a de novo $16.8 \mathrm{~kb}$ deletion that includes most of OFD1. In five further cases (17\%) we identified de novo or inherited recessive or X-linked variants in plausible candidate genes, which require additional validation to determine pathogenicity. Our diagnostic yield of $10 \%$ is comparable to, and supplementary to, the diagnostic yield of existing microarray testing for large chromosomal rearrangements and targeted CNV detection. The de novo nature of these events could enable couples to be counseled as to their low recurrence risk. This study outlines the way for a substantial improvement in the diagnostic yield of prenatal genetic abnormalities through the application of next-generation sequencing.

\section{INTRODUCTION}

Fetal abnormalities detected using ultrasound examination range from minor findings (i.e. dysmorphic facial features) to major, potentially lethal, multisystem abnormalities. The underlying etiology is diverse and includes environmental and genetic factors. Congenital structural anomalies affect $2.2 \%$ of births in UK and Wales (1), and the prevalence of structural fetal anomalies is likely to be even higher than this, because a proportion of these pregnancies are associated with fetal loss.
Typically, the genetic etiology of fetal abnormalities is investigated (prenatally or postnatally) by a combination of karyotyping, fluorescence in situ hybridization (FISH) and array-based comparative genomic hybridization (aCGH). Conventional full karyotyping may detect numerical as well as unbalanced and balanced structural rearrangements $>5-10 \mathrm{Mb}$. Submicroscopic chromosomal anomalies may be diagnosed using FISH, but this is highly limited in being a 'targeted' approach, requiring knowledge that a specific karyotypic rearrangement is associated with a specific malformation. The

\footnotetext{
*To whom correspondence should be addressed at: School of Clinical and Experimental Medicine (Birmingham Centre for Women's and Children's Health), College of Medical and Dental Sciences, University of Birmingham, Edgbaston, Birmingham, B15 2TT, UK. Tel: +44 1216272778; Fax: +441216236875; Email: m.d.kilby@bham.ac.uk

(C) The Author 2014. Published by Oxford University Press.

This is an Open Access article distributed under the terms of the Creative Commons Attribution License (http://creativecommons.org/licenses/by/3.0/), which permits unrestricted reuse, distribution, and reproduction in any medium, provided the original work is properly cited.
} 
popularity of aCGH in prenatal diagnostics is increasing. The sensitivity for detection is dependent upon the platform chosen and the indication for testing. A recent systematic review reported a prenatal detection rate of $10 \%$ (95\% confidence interval (CI), 8-13) above conventional karyotyping in fetuses with structural malformations (2). This is consistent with studies estimating the diagnostic rate of aCGH in fetuses with structural abnormalities as $6-10 \%(3,4)$. However, aCGH is limited to the detection of copy-number variants (CNVs) of $>10-100 \mathrm{~kb}$ in specifically targeted regions.

Next-generation sequencing (NGS) is becoming an invaluable tool not just for disease gene discovery in a research setting, but also for clinical diagnostics. The diagnostic yield of exome sequencing for patients with Mendelian diseases is $25 \%$ (5), suggesting that it might complement conventional prenatal diagnostic techniques. NGS could improve prenatal diagnostic yield by identifying pathogenic genetic variants that are below the resolution of aCGH platforms in current clinical use, and by localizing breakpoints of cytogenetically balanced chromosomal rearrangements to individual genes (6). To date, two studies have described the clinical use of NGS to make a diagnosis for a single fetus $(5,6)$, and one study of a larger cohort reported low-coverage NGS for identifying prenatal aneuploidy and unbalanced chromosomal rearrangements (7). In our proof-of-principle study of 30 fetuses and neonates with structural anomalies first identified by ultrasound, we illustrate the power of whole-exome sequencing for identifying variants (single-nucleotide variants (SNVs), indels and CNVs) that potentially cause abnormal fetal development.

\section{RESULTS}

\section{Exome sequencing}

Exome sequencing in 30 fetuses and neonates with a diverse range of structural abnormalities diagnosed at prenatal ultrasound, along with their parents, was performed (a total of 86 individuals). The mean depth of coverage of the targeted coding regions was $103 \times$. A mean of $92.7 \%$ of bases in the targeted coding regions were covered by at least 10 reads (Supplementary Material, Table S1 and Figs S1-S3). No parental phenotypic abnormalities were reported that might be related to the fetal abnormalities, suggesting dominant inheritance is unlikely. We therefore evaluated different classes of potentially pathogenic, rare coding variants under dominant de novo, recessive and $\mathrm{X}$-linked modes of inheritance, through systematic manual curation of the existing literature, to classify variants into one of three categories: highly likely to be causal, possibly causal or unknown. For the three non-sporadic cases (the siblings F27 and F33, and F2, who has a similarly affected sibling not included in this study), all of which are female, we consider a recessive mode of inheritance most likely. We nevertheless investigated all the mutation classes described above.

\section{De novo SNVs and indels}

We identified potential de novo SNVs and indels with high sensitivity, and inevitably low specificity, yielding a list of 77 candidate de novo coding or splicing variants (mean $=2.6$ per fetus, range $=0-5$ ). We attempted to validate all of these by capillary sequencing of whole genome amplified genomic DNA, irrespective of their predicted functional consequence. We validated 34 as being truly de novo (Table 1 and Supplementary Material, Table S2). This is a mean of 1.13 per fetal exome (range $0-4$ ), which is within the expected range from the known germline mutation rate and NGS of other disease cohorts (8-11). These mutations include identical PPFIBP2 mutations in the monozygotic twins F3 and F16, with the result that there are 33 independent de novo variants.

The expected percentage of de novo variants in coding or splicing sequence that are synonymous is $29 \%$ (12), however, we observed that only three $(9 \%)$ of the 33 validated independent de novo variants were synonymous, with 26 being nonsynonymous, three nonsense and one in a splice site. Thus the proportion of validated de novo mutations that are predicted to have a functional consequence of the encoded protein is significantly enriched over what would be expected by chance $(P=0.007)$, suggesting that an appreciable subset of these functional mutations is likely to be pathogenic. Of the non-synonymous variants, $10(38.5 \%)$ are predicted to be probably damaging by Polyphen, $13(50 \%)$ are predicted to be deleterious by sorting intolerant from tolerant, and seven (26.9\%) are predicted to be damaging by both methods (Supplementary Material, Table S2). We found suggestive evidence of mosaicism for two of the de novo mutations: c. $313 \mathrm{G}>\mathrm{C} \quad(\mathrm{p} .105 \mathrm{E}>\mathrm{Q})$ in $P A R D 3 B$ (ENST00000349953) in F9, and c.2921G $>$ T (p. 974C $>$ F) in SEC31B (MIM 610258, ENST00000370345) in F33 (Supplementary Material, Table S2).

Two of the de novo missense variants are highly likely to be pathogenic. In F23, a male fetus with features consistent with lethal skeletal dysplasia, we found c.1118A $>\mathrm{G}$ (p.373Y > C) in FGFR3 (MIM 134934 (http://www.omim.org/ ), ENST00000440486 (http://www.ensembl.org/)). FGFR3 is a negative regulator of bone growth, missense mutations in which cause a wide range of skeletal dysplasias. p. $373 \mathrm{Y}>\mathrm{C}$ is known to cause thanatophoric dysplasia (13), giving high confidence that this is the causative mutation in F23.

In F20, a male fetus with increased nuchal translucency $(>3.5 \mathrm{~mm})$, tricuspid regurgitation and abnormal legs and feet with an extended posture and bilateral talipes equinovarus anomaly, we found c.3490G $>\mathrm{T}(\mathrm{p} .1164 \mathrm{G}>\mathrm{C})$ in COL2A1 (MIM 120140, ENST00000380518). COL2A1 mutations can cause type II collagenopathies, some of which include heart and limb defects $(14,15)$. Importantly, p.1164G $>C$ is a glycine to non-serine substitution in the triple helical domain of COL2A1, which is a particularly damaging class of mutations (16), although p.1164G $>C$ has not previously been reported.

We found two possibly pathogenic de novo missense variants in F6, a female fetus with abdominal situs inversus, cardiac malposition of the great arteries and multiple ventricular septal defects. These features are consistent with Ivemark's syndrome (MIM 208530), the molecular basis of which is unknown. First, we found c. 2747G >A (p.916R >Q) in NF1 (MIM 613113, ENST00000456735). Variants in NF1 can be associated with congenital heart defects, and knocking down either zebrafish orthologue causes cardiovascular defects $(17,18)$. Furthermore, substitution of this particular amino acid has been previously proposed to be pathogenic (19). Second, we found c.1555C $>\mathrm{T}$ (p.519R >*) in SMARCC2 (MIM 601734, ENST00000267064). This encodes a SWItch/Sucrose NonFermentable-related 
Table 1. Candidate genes identified in 27 fetuses with structural abnormalities

\begin{tabular}{|c|c|c|c|c|c|c|}
\hline ID & Gender & $\begin{array}{l}\text { Genes with } \\
\text { de novo mutations }\end{array}$ & $\begin{array}{l}\text { Genes with inherited autosomal mutations } \\
\text { (recessive, compound heterozygous) }\end{array}$ & $\begin{array}{l}\text { Genes with inherited } \\
\text { autosomal mutations } \\
\text { (recessive, homozygous) }\end{array}$ & $\begin{array}{l}\text { Genes with inherited mutations on } \\
\mathrm{X} \text { chromosome (hemizygous) }\end{array}$ & Genes in CNVs \\
\hline F1 & Male & - & HEPHL1; PRKDC; ZNF44 & - & BCORL1; FAM47A; KCNEIL; MAGEA6; ZCCHC12 & - \\
\hline F2 & Female & GRIN2A & FAM 83E; KIAA1239; KIAA1755; LAMA5; MIA3 & - & & - \\
\hline $\mathrm{F}^{\mathrm{a}}$ & Male & PPFIBP2 & C16orf91; C9orf79; CCDC144NL; NHSL1 & - & CCDC22; SHROOM2 & {$[H 2 B F M ; H 2 B F W T]$} \\
\hline F5 & Male & & $D L C 1 ; T T N$ & - & $\begin{array}{l}\text { FAM70A; FTHL17; GPR112; PCDH19; RBMXL3; } \\
\quad \text { WDR44 }\end{array}$ & - \\
\hline F6 & Female & Cllorf41; NF1; SMARCC2; ZHX3 ${ }^{\mathrm{b}}$ & FAM188B; RELN; RERE & $A X L$ & - & - \\
\hline F7 & Female & UNC80; WFDC8 & MUC16; TSC22DI; TTN & - & - & - \\
\hline F8 & Male & CD244 & LY75-CD302; TTN; WDR59 & - & $P L X N B 3 ; R B B P 7 ;$ SRPX2 & - \\
\hline F9 & Male & $P A R D 3 B$ & $\begin{array}{l}\text { ABCA13; COL6A6; GNAS; KIAA1462; MUC17; } \\
\text { SRRM2; TRPM } 8\end{array}$ & - & $A T P 2 B 3 ; C C D C 22$ & - \\
\hline F10 & Female & $A T P 6 V 1 B 2 ; S E M A 4 D^{\mathrm{c}}$ & C19orf $28 ;$ CDHRI; DNAH10; MACFI & - & - & - \\
\hline F11 & Male & - & REST & - & CITED $1 ; M X R A 5 ; N R O B 1$ & - \\
\hline F12 & Female & - & FRGIB; TTN; ZNF451 & - & - & - \\
\hline F13 & Male & - & FRASI; SPTBN5; TPO & - & $A L G 13 ; D D X 26 B ; M A P 7 D 3 ; T L R 7$ & - \\
\hline F14 & Female & KCTD $8 ;$ STX12 & ADNP; ANO7; CENPF; TDRD6 & - & - & {$[G P M 6 B ; O F D I]$} \\
\hline F15 & Female & DOCK1 & ABLIM $3 ;$ VCAN & - & - & - \\
\hline $\mathrm{F} 16^{\mathrm{a}}$ & Male & PPFIBP2 & C16orf91; C9orf79; CCDC144NL; NHSL1 & - & SHROOM 2 & - \\
\hline F17 & Female & - & ABCA $3 ;$ AKAP11; DEPDC1; PAFAH2; POM121C & - & - & - \\
\hline F18 & Male & $A B C B 9 ; F A M 3 D$ & $P C C B ; T T N ; Z F H X 3$ & - & $\begin{array}{l}\text { CXorf57; DUSP } 21 ; F 9 ; \text { FOXR2; HS6ST2; NKAP; } \\
\quad \text { RBMX2 }\end{array}$ & - \\
\hline $\mathrm{F} 19^{\mathrm{e}}$ & Male & DNAJC13 ${ }^{\mathrm{b}} ; N L R P 1 ; P A R D 3 B$ & $\begin{array}{l}\text { AHNAK2; C20orf } 90 ; \text { CD163L1; DNAH1; DNAH5; } \\
\quad \text { DNAH6; FSTL4; PHLPP2 }\end{array}$ & $A D A D 2 ; P C N T$ & COL4A6; GYG2; PNMA3; SATL1; SHROOM2 & {$[S S X 3 ; S S X 4 ; S S X 4 B]$} \\
\hline F20 & Male & COL2A1 & CHD7; EPB41L2; GPR98; VPS13D & - & FAM58A; MTCP1NB; PLXNA3; SLC10A3 & - \\
\hline F21 & Male & - & CACNA1H; PKHDI & KIF26A & $\begin{array}{l}\text { ARMCX2; EDA2R; HTATSF1; MAP7D } 3 ; \text { MTMR } 8 ; \\
\quad \text { MXRA5 }\end{array}$ & - \\
\hline F22 & Male & TACR2 & DECRI; DUOXA1; NEB; VPSI3C & PCDHB7 & $M A P 7 D 3$ & - \\
\hline F23 & Male & FGFR3 & Clorf129; SHANK2; TTN & GFM2 & $M A P 3 K 15 ; M A P 7 D 3$ & - \\
\hline F25 & Male & PNLIPRPI; SMARCCI & $H S P G 2 ; I Q G A P 3$ & - & BCOR; RAB40A; USP 26 & - \\
\hline F26 & Male & KDM5B; STAU2 & GNRHR2 & - & HTATSF $1 ;$ MTMRI; PIR & - \\
\hline $\mathrm{F} 27^{\mathrm{d}}$ & Female & C2orf40; INSC & - & - & - & - \\
\hline F28 & Female & PPP6R1 & CYP24A1; KIAA1109; KIAA1609; SLC39A11 & - & - & - \\
\hline F29 & Female & - & $\begin{array}{l}\text { ABCA13; MCF2L2; NLRP12; POM121C; } \\
\quad T T N ; Z N F 831\end{array}$ & TTN & - & - \\
\hline F31 & Female & $F M N L 3$ & $F A H$ & - & - & - \\
\hline F32 & Female & & - & - & - & - \\
\hline $\mathrm{F} 33^{\mathrm{d}}$ & Female & $S E C 31 B ; E G F L 6^{\mathrm{b}}$ & $A G R N ; N U D T 19$ & - & - & - \\
\hline
\end{tabular}

Square brackets contain genes in a single CNV. Variant information is detailed in the Supplementary Material, Appendix for the de novo SNPs and indels (Supplementary Material, Table S2), inherited recessive and X-linked SNPs and indels (Supplementary Material, Table S3) and CNVs (Supplementary Material, Table S4 and Supplementary Material, Fig. S4). 'Possibly causal' genes are indicated in orange and 'highly likely to be causal' genes in red.

${ }^{\mathrm{a}}$ Monozygotic twins.

Synonymous de novo variant.

'We looked for inherited, rare, functional, 'second hit' variants in genes in which we found de novo mutations and found only one: a 9:92006277C $>$ G heterozygous, maternally inherited missense variant in SEMA4D in F10,

${ }^{\mathrm{d}}$ Siblings.
${ }^{T}$ This fetus is of Indian ancestry, whereas the majority of the cohort is of European ancestry. This is likely to explain why there are so many apparently rare inherited candidate variants in this case. 
chromatin regulator. Variants within several genes that encode components of the same protein complex can cause developmental disorders $(20,21)$.

Two of the unrelated fetuses had de novo missense mutations in $P A R D 3 B$. F9, a male fetus with a complex brain malformation and unilateral talipes equinovarus had the $P A R D 3 B$ mutation c. $313 \mathrm{G}>\mathrm{C}$ (p.105E $>$ Q). F19, a male with an atrial septal defect, esophageal atresia and a unilateral facial cleft had the mutation c.731G $>$ A (p.244R $>$ Q). The likelihood of two functional de novo mutations in a gene of the size of $P A R D 3 B$ occurring by chance in unrelated probands in a cohort of this size is small $\left(P=3.1 \times 10^{-6}\right.$, which does not reach the Bonferronicorrected significance threshold for testing of all genes of $P=$ $2.5 \times 10^{-6}$ ). De novo PARD3B mutations have not been reported in other larger sequencing studies suggesting that $P A R D 3 B$ does not have an unusually high mutation rate $(9,11)$. $P A R D 3 B$ encodes partitioning defective 3 homolog $\mathrm{B}$ (Par3b), which is involved in cell polarization (22). It has a paralogue, $P A R D 3$, which has a role in various developmental processes including neurogenesis (23). Homozygous mouse knockouts for Par3 are embryonic lethal and have growth retardation, heart and brain defects and short tails (24), and zebrafish Pard3 knockdowns have hydrocephalus (23). The overlap between phenotypes resulting from knockdown of $P A R D 3$ and the phenotypes in F9 and F19 is interesting, however we judged that the current knowledge of the function of $P A R D 3 B$ is insufficient to categorize the mutations identified in our cohort as being possibly causal.

De novo variants in genes known to be involved in developmental disease were not necessarily classified as possibly causal, where the phenotype of the fetus did not overlap sufficiently with previously reported phenotypes. For example, the de novo missense mutation c. $4354 \mathrm{C}>\mathrm{T}$ ( $\mathrm{p} .1452 \mathrm{R}>\mathrm{C}$ ) in GRIN2A (MIM 138253, ENST00000461292) was found in F2, a female with atrioventricular septal defect (AVSD), hepatic dysfunction, polydactyly, panhypopituitarism and brain injury. GRIN2A mutations can cause seizures and intellectual disability, and are highly unlikely to be the cause of the multiple structural malformations seen in F2 (25). Supporting this assertion is the fact that this individual had an older sibling with a similar phenotype, making de novo variants an unlikely cause of disease.

\section{Inherited recessive and $\mathrm{X}$-linked $\mathrm{SNVs}$ and indels}

We detected a mean of 21444 high-quality coding SNVs and indels per individual (Supplementary Material, Table S1). We identified potentially relevant inherited recessive and X-linked variants (SNVs and indels) by filtering for rare (minor allele frequency $<1 \%$ ), functional, hemizygous, homozygous or compound heterozygous mutations. This identified a mean of 5.3 candidate genes per fetus (range of $0-15$ ) with a total of 139 different candidate genes across the 30 fetuses, containing 269 rare functional variants. Of these variants, 262 are missense, four are frameshift and three are nonsense (Table 1 and Supplementary Material, Table S3).

Inherited variants in five of the fetuses are possibly causal. These mutations have been verified by Sanger sequencing of whole genome amplified genomic DNA (data not shown). In $\mathrm{F} 1$, a male fetus with multiple abnormalities including limb defects, craniofacial defects, anogenital defects, heart defects, a tracheal esophageal fistula and renal agenesis, we found the compound heterozygous mutations c.9598C $>\mathrm{T}(\mathrm{p} .3200 \mathrm{P}>\mathrm{S})$ and c. $1420 \mathrm{G}>\mathrm{T}(\mathrm{p} .474 \mathrm{~V}>\mathrm{F}$ ) in PRKDC (MIM 600899, ENST00000338368). PRKDC encodes DNA-PKcs, which, in complex with $\mathrm{Ku}$, is required for the DNA double-strand break repair mechanism non-homologous end-joining. In humans, PRKDC mutations can cause severe combined immunodeficiency due to defective $\mathrm{V}(\mathrm{D}) \mathrm{J}$ recombination, and severe cases can also have abnormalities of the brain, face, limbs and anogenital organs (26).

In F5 who had cardiac truncus arteriosus, type B interruption of the aortic arch and pyloric stenosis, we found the compound heterozygous mutations c. $2189 \mathrm{G}>\mathrm{A}(\mathrm{p} .730 \mathrm{R}>\mathrm{Q})$ and c.721C $>\mathrm{G}$ (p.241P > A) in DLC1 (MIM 604258, ENST00000276297). Homozygous $D L C l$ knockout mice are embryonic lethal with deformities of brain and heart (27). In F6, whose phenotype has been described, we found the compound heterozygous mutations c. $4264 \mathrm{G}>\mathrm{A}(\mathrm{p} .1422 \mathrm{~V}>\mathrm{M})$ and c.3686G $>\mathrm{A}(\mathrm{p} .1229 \mathrm{R}>\mathrm{Q})$ in RERE (MIM 605226, ENST00000337907). Homozygous mouse knockouts develop asymmetrically and have cardiovascular defects, while homozygous zebrafish mutants have various defects including abnormal fins and brains (28-30). In total we have identified two genes with de novo variants and one gene with inherited variants that could possibly account for the phenotype in F6. It is not possible to say which is most likely to be causative, as none of the candidate genes are known to harbor variants that cause the exact phenotype reported here. One possibility is that multiple variants contribute to this multisystemic phenotype, as has been reported in other exome sequencing studies of rare disease (5).

F10 had fetal akinesia syndrome probably caused by neuroaxonal dystrophy. We found the compound heterozygous mutations c. $5323 \mathrm{G}>\mathrm{A}(\mathrm{p} .1775 \mathrm{E}>\mathrm{K})$ and c.8626A $>\mathrm{G}(\mathrm{p} .2876 \mathrm{I}>\mathrm{V})$ in MACF1 (MIM 608271, ENST00000372925), which encodes cytoskeletal protein microtubule-actin cross-linking factor 1. Knockout of the mouse orthologue causes defects in axonal extension (31). Finally, F13 had multiple abnormalities including a multicystic-dysplastic kidney, distorted ribs and spine, brain defects and bilateral talipes equinovarus. Here we noted the compound heterozygous missense mutations c.1918C $>\mathrm{T}$ (p.640R > C) and c.5205C > A (p.1735H > Q) in FRAS1 (MIM 607830, ENST00000264895), in which mutations are associated with Fraser syndrome (MIM 219000). FRAS1 has a role in renal development (32), and knockout mice have severely defective kidney development, along with syndactyly (33). Homozygous zebrafish mutants have malformed fins and pharyngeal pouches, suggesting a role for FRAS1 in skeletal development (34).

F19 has a high number of inherited, apparently rare variants (Table 1). F19 is of Indian ancestry, whereas the majority of the cohort is of European ancestry. It is likely therefore that a subset of the apparently rare variants that we have identified in F19 are in fact common in this population, but we have not been able to identify them as such due to an underrepresentation of individuals of Indian ancestry in the databases we used to filter the variants.

\section{CNVs}

CNVs from the exome data were denoted using the CoNVex program. We identified three rare, high-quality CNVs (one 
deletion and two duplications) under de novo, inherited recessive or X linked models (Table 1 and Supplementary Material, Table S4 and Supplementary Material, Fig. S4).

One of these was the de novo $21 \mathrm{~kb}$ deletion g.13770686 13791294del on Xp22.2 found in F14, a female fetus with ventriculomegaly and agenesis of the corpus callosum, This CNV removes most of the gene OFDI (MIM 300170). Mutations in OFD1 cause orofaciodigital syndrome 1 (MIM 311200), which in $40 \%$ of cases includes central nervous system anomalies, including the absence of the corpus callosum (35). This deletion has been confirmed by aCGH, is highly likely to be pathogenic, and has a low $(<1 \%)$ recurrence risk.

\section{DISCUSSION}

In this study, we performed exome sequencing of 30 fetuses or newborns (along with their parents) with diverse congenital structural abnormalities identified by fetal (prenatal) ultrasound. We identified an average of one candidate gene with a de novo functional variant and five candidate genes with inherited functional variants per fetus. Variants that are highly likely to be causal were noted in three cases (10\%). Variants that are possibly causal but require further confirmatory genetic and functional studies were noted in a further five cases $(17 \%)$.

Proof of principle of prenatal NGS for diagnosis of aneuploidy and chromosomal rearrangements has been established $(6,7)$. Subsequently, four fetuses were included in a cohort of 250 patients with Mendelian disorders who were exome sequenced, of which one was successfully diagnosed (5). There are other cases in which postnatal exome sequencing of cohorts with specific diseases first manifesting in the prenatal period have yielded diagnoses $(36,37)$. Our study of a cohort of 30 fetuses with diverse abnormalities has allowed us to estimate the current diagnostic yield of exome sequencing for fetal abnormalities as being broadly equivalent to aCGH, which is $\sim 10 \%(2-4)$. However, due to the small cohort size our estimate of diagnostic yield has a broad CI. While some of our samples were obtained from neonates subsequent to live birth, the abnormalities of the entire cohort were first identified at the prenatal stage, so our estimated diagnostic yield is equivalent to what would be expected for entirely prenatal testing. Larger studies would not only provide a more accurate diagnostic rate, but would allow for additional interesting analyses such as stratification of the cohort on the basis of phenotypic severity, which could aid in identification of pathogenic variants.

The primary challenge faced in this study that is shared across all such studies was interpreting the clinical significance of the candidate mutations we identified. There is therefore considerable potential for increase of the diagnostic yield once understanding of the genetic architecture of developmental disorders has improved. However, an additional factor here is that for most genes where disruptive genetic variants can cause developmental disorders, our understanding of the phenotypic consequences of this variation is limited to postnatal observations that are often not detectable by prenatal ultrasound investigations (e.g. intellectual disability in the absence of structural brain malformations). This could explain why our diagnostic yield is lower than that of similar studies of postnatal disorders (5). Moreover, given that postnatal observations are inherently subject to survival bias, it may be that in the prenatal setting more severe phenotypes can be observed for the same variants (38).

The de novo occurrence of the three clearly pathogenic variants identified in this prenatal study could have enabled the parents to be counseled that the risk of recurrence in subsequent pregnancies is low, and highlights the value to families of receiving a clear genetic diagnosis. In this study, none of the results from the exome sequencing were relayed to patients. Our finding that most currently diagnostic variants from exome sequencing arise de novo is in agreement with recent studies of patients with intellectual disability, many of whom also have congenital structural abnormalities $(9,11)$. Interestingly, only one of the three variants that are highly likely to be causal would have been detected by aCGH alone (the $\mathrm{CNV}$ overlapping OFD1 in F14), and only one could have been suspected as a candidate gene from the ultrasound findings alone ( $F G F R 3$ variants in F23 with thanataphoric dysplasia).

Clinical implementation of exome sequencing for prenatal diagnosis of structural anomalies identified by ultrasound promises to improve management of pregnancy and enable more informative counseling to parents. However, it also poses several challenges. First, the analytical strategy we adopted was labor intensive and not scalable. Clinical implementation would require the development of large-scale and rapid analytical and interpretation pipelines, as well as rigorous health economic assessments. Second, to facilitate interpretation of prenatal variation, it will be necessary to develop a vastly more detailed knowledge base on the genetic causes of prenatal developmental disorders. Finally, the sequencing data in this study were not produced within a timeframe that would have allowed the parents to take it into account when making a decision about the outcome of the pregnancy being tested. This would of course be the ideal scenario, and feasibility in principle has been demonstrated (6).

In conclusion, our study shows that exome sequencing is a promising method with which to identify genetic variants that cause structural fetal abnormalities and thereby improve the clinical management of pregnancies and better inform the reproductive decisions of affected families. The challenges to clinical implementation are considerable, but surmountable, and we envisage that exome sequencing, initially in a clinical research context, will be an important addition to prenatal genetic diagnostics in the near future.

\section{MATERIALS AND METHODS}

\section{Cohort}

The National Research Ethics Service Committee (West Midlands - Staffordshire, UK) approved this study (REC reference 09/H1203/74). We recruited a cohort of 33 fetuses and their nonconsanguineous parents seen for prenatal diagnosis at the Fetal Medicine Centre Birmingham Women's Foundation Trust, UK. This is a subgroup (12\%) of a larger cohort described previously (2). The median maternal age at diagnosis was 31 years (range 18-39). We included women who had a fetus with a structural anomaly suspected at their routine ultrasound scan at $11-$ 14 weeks or $18-20$ weeks gestation. The women were subsequently rescanned at a tertiary referral center and the diagnoses confirmed. The median gestational age at confirmation of diagnosis was 21 weeks (range 11-35). 
Table 2. Sampled tissue and outcomes

\begin{tabular}{lllc}
\hline Pregnancy outcome & Number (and percent of cohort) & Sampled tissue & Number (and percent of cohort) \\
\hline Termination or miscarriage & $19(63 \%)$ & Placenta & $12(40 \%)$ \\
& & Blood & $4(13 \%)$ \\
& & Chorionic villus sample & $2(7 \%)$ \\
Live birth & $11(37 \%)$ & Other tissues (fetal liver/lung) & $1(3 \%)$ \\
& & Cord blood & $6(20 \%)$ \\
& & Chorionic villus sample & $2(7 \%)$ \\
& & Placenta (postnatally) & $1(3 \%)$ \\
& Cultured amniocytes & $1(3 \%)$ & $1(3 \%)$ \\
\hline
\end{tabular}

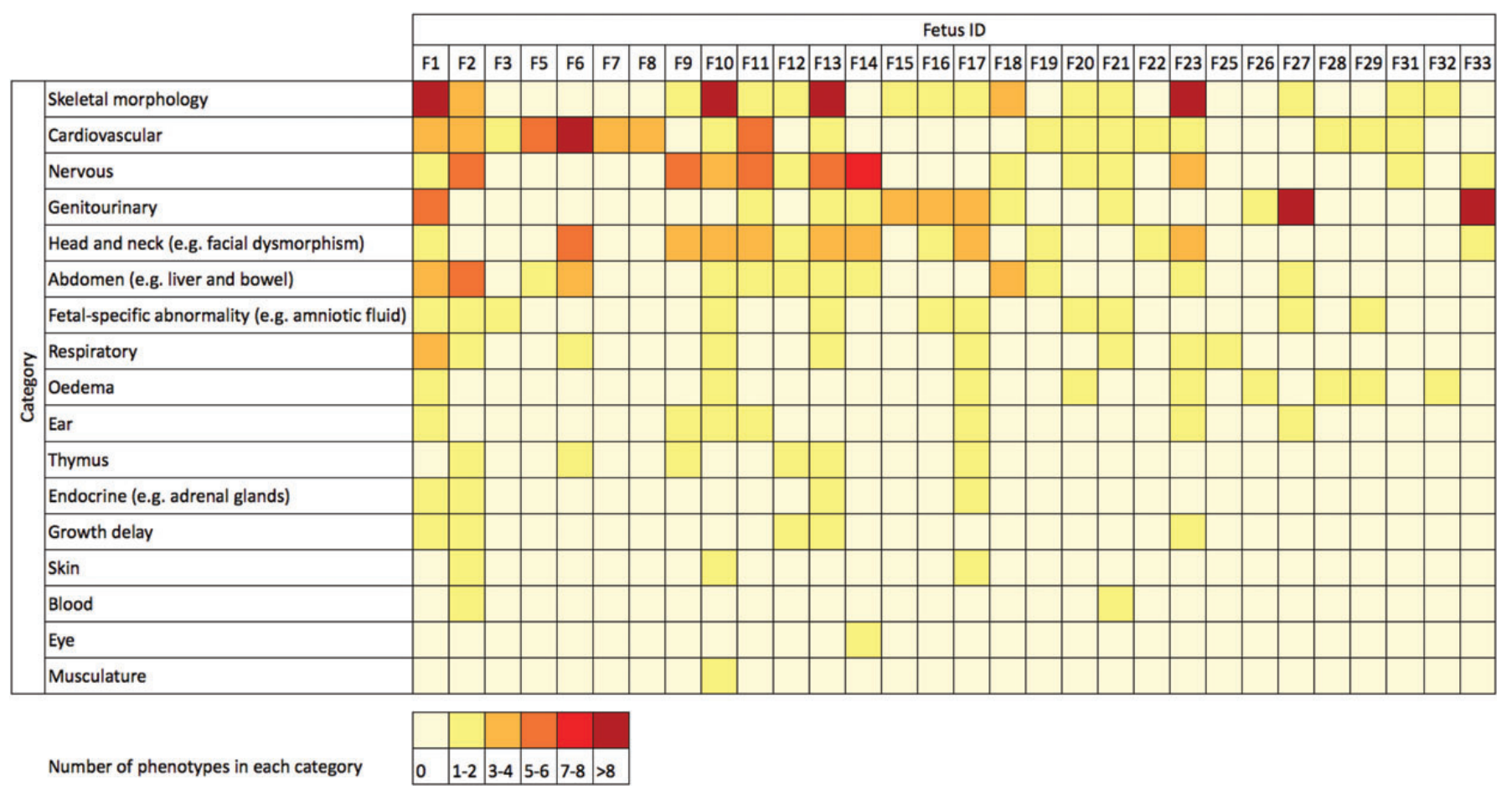

Figure 1. Matrix of phenotypes in the cohort. For each fetus (F1-F33), the color indicates the number of observed phenotypes that are in each category of phenotypes. For example, F1 has more than eight separate abnormalities of skeletal morphology. The categories are modified higher order HPO terms, and the data come from ultrasound scans, postmortem reports or pediatric follow-up. The phenotype of each fetus is detailed in the Supplementary Material, Appendix.

DNA of affected fetuses or neonates was obtained from various sources. Where a pregnancy was terminated or miscarried, fetal DNA was most commonly collected from placental tissue. Where a live birth occurred, neonatal DNA was usually collected from cord blood at delivery (Supplementary Material, Appendix and Table 2). Parental DNA was obtained from venous blood. In this article, the participants are identified by their trio number (1-33) prefaced by $\mathrm{F}$ for the fetus, $\mathrm{M}$ for the mother and P for the father. There are two exceptions to this, as the cohort includes two sets of related fetuses. F3 and F16 are monozygotic twins; therefore the parents of F16 are M3 and P3. F27 and F33 are siblings; therefore the parents of F33 are M27 and P27. F2 has an older sibling with a similar phenotype, who is not included in this study. The remaining fetuses are sporadic cases, and none of the parents had phenotypic abnormalities that were likely to be related to that of the fetuses.
Prior to inclusion in this study the G-band karyotypes of all cases except $\mathrm{F} 33$ were confirmed as normal, and a low-resolution targeted $1 \mathrm{Mb}$ BAC array (BlueGnome) did not demonstrate any likely pathological CNVs. For F33, a combination of quantitative fluorescent PCR and multiplex ligation-dependent probe amplification revealed no aneuploidy, or deletions or duplications in the subtelomeric regions. For the 95 samples, we obtained a mean of $3 \mu \mathrm{g}$ DNA, but 12 samples had less than the required $0.2 \mu \mathrm{g}$ DNA for exome sequencing. We attempted to sequence all samples, but exome sequencing failed due to insufficient DNA in trios 4, 24 and 30, leaving a total cohort of 26 trios and two quads (couple with two affected fetuses) $(91 \%, 86$ samples).

The fetuses and neonates had a wide range of structural abnormalities. In addition to those identified by detailed ultrasound scan and investigation in a tertiary Fetal Medicine Centre, 


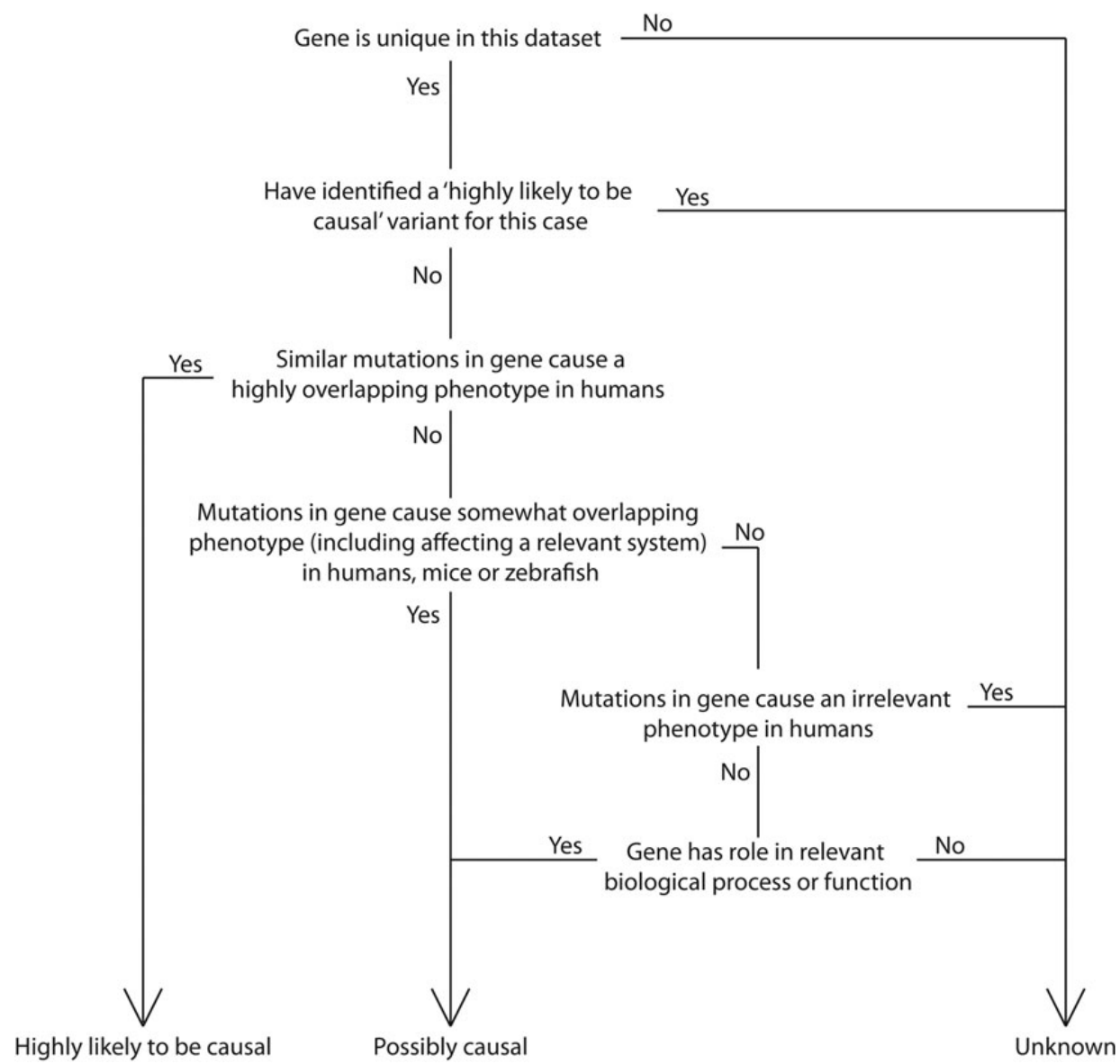

Figure 2. Decision tree for prioritizing candidate genes into three categories. Data from OMIM, DDG2P, BioGPS, NHGRI GWAS catalog, IKMC, ZFIN and PubMed were used, where available.

phenotypic information was collected for a subset of cases from postmortem reports by perinatal pathologists $(n=12)$, or pediatric follow up $(n=7)$. Phenotype data were converted to Human Phenotype Ontology (HPO) terms (Fig. 1 and Supplementary Material, Appendix) (39).

\section{Exome sequencing and candidate variant identification}

For detailed methodology see Supplementary Material, Appendix. In brief, DNA samples were exome sequenced using a SureSelect All Exon capture kit (Agilent, Wokingham, UK) followed by paired-end sequencing ( 75 bp reads) on the HiSeq platform (Illumina, Saffron Walden, UK). Reads were mapped to the reference human genome GRCh37_hs37d5. Duplicate reads were removed and reads were realigned around potential indels. We called variants using SAMtools, GATK and Dindel. The EGA ID for the exome sequencing data is EGAS00001000167 (https://www.ebi.ac.uk/ega/).

To identify de novo mutations, we used De Novo Gear pipeline version 0.6.2 (40). We considered rare, high-quality variants in protein-coding exons. To identify inherited recessive and X-linked SNVs and indels, we first merged the VCFs from the three variant callers for each individual, and then we identified genes harboring rare, high-quality, functional variants (predicted protein consequences were essential splice site, stop gained, frameshift coding, non-synonymous, stop lost) under the different plausible modes of inheritance (recessive or $\mathrm{X}$-linked). To discover CNVs from the exome data, we used CoNVex (ftp://ftp.sanger.ac.uk/pub/users/pv1/CoNVex/). We filtered out $\mathrm{CNVs}$ that were low confidence, overlapped common CNVs, did not overlap protein-coding genes or did not fit with the expected mode of inheritance (de novo, inherited recessive or X-linked).

\section{Classification of variants}

To classify the variants we annotated each candidate gene with functional information (where available) from OMIM (http://www.omim.org/), DDG2P (http://decipher.sanger.ac. uk/ddd/ddd_genes), BioGPS (biogps.org), NHGRI GWAS catalog (http://www.genome.gov/gwastudies/), IKMC (http:// www.knockoutmouse.org/), ZFIN (http://zfin.org/) and PubMed (http://www.ncbi.nlm.nih.gov/pubmed). We used a decision tree to classify each variant as being highly likely to be causal, possibly causal but requires further genetic or functional confirmatory studies, or unknown (Fig. 2). 


\section{SUPPLEMENTARY MATERIAL}

Supplementary Material is available at $H M G$ online.

\section{ACKNOWLEDGEMENTS}

We thank all the staff of the Fetal Medicine Centre at Birmingham Women's Foundation Trust and Drs Philip Cox and Tamas Martin (Department of Perinatal Pathology) for discussion relating to fetal phenotypes. We are grateful to the families who participated in this study, and to Mr Daniel King, Dr Saeed Al-Turki, Dr Sarah Lindsay, Dr Don Conrad, Fiona Togneri and Georgina Hall for assistance with data collection and analyses.

Conflict of Interest statement. The authors declare no conflicts of interest.

\section{FUNDING}

This work was supported by the Wellcome Trust (WT098051); and SPARKS - The Children's Medical Research Charity (09RTF07 to S.C.H.). Funding to pay the Open Access publication charges for this article was provided by the Wellcome Trust (WT098051).

\section{REFERENCES}

1. Springett, A. and Morris, J.K. (2010) Congenital anomaly statistics. England and Wales. London: British Isles Network of Congenital Anomaly Registers.

2. Hillman, S.C., McMullan, D.J., Hall, G., Togneri, F.S., James, N., Maher, E.J., Meller, C.H., Williams, D., Wapner, R.J., Maher, E.R. et al. (2013) Use of prenatal chromosomal microarray: prospective cohort study and systematic review and meta-analysis. Ultrasound Obstet. Gynecol., 41, 610-620.

3. Valduga, M., Philippe, C., Bach Segura, P., Thiebaugeorges, O., Miton, A., Beri, M., Bonnet, C., Nemos, C., Foliguet, B. and Jonveaux, P. (2010) A retrospective study by oligonucleotide array-CGH analysis in 50 fetuses with multiple malformations. Prenat. Diagn., 30, 333-341.

4. Wapner, R.J., Martin, C.L., Levy, B., Ballif, B.C., Eng, C.M., Zachary, J.M., Savage, M., Platt, L.D., Saltzman, D., Grobman, W.A. et al. (2012) Chromosomal microarray versus karyotyping for prenatal diagnosis. N. Engl. J. Med., 367, 2175-2184.

5. Yang, Y., Muzny, D.M., Reid, J.G., Bainbridge, M.N., Willis, A., Ward, P.A., Braxton, A., Beuten, J., Xia, F., Niu, Z. et al. (2013) Clinical whole-exome sequencing for the diagnosis of Mendelian disorders. N. Engl. J. Med., 369, 1502-1511.

6. Talkowski, M.E., Ordulu, Z., Pillalamarri, V., Benson, C.B., Blumenthal, I., Connolly, S., Hanscom, C., Hussain, N., Pereira, S., Picker, J. et al. (2012) Clinical diagnosis by whole-genome sequencing of a prenatal sample. N. Engl. J. Med., 367, 2226-2232.

7. Dan, S., Chen, F., Choy, K.W., Jiang, F., Lin, J., Xuan, Z., Wang, W., Chen, S., Li, X., Jiang, H. et al.. (2012) Prenatal detection of aneuploidy and imbalanced chromosomal arrangements by massively parallel sequencing. PLoS One, 7, e27835.

8. Awadalla, P., Gauthier, J., Myers, R.A., Casals, F., Hamdan, F.F., Griffing, A.R., Cote, M., Henrion, E., Spiegelman, D., Tarabeux, J. et al. (2010) Direct measure of the de novo mutation rate in autism and schizophrenia cohorts. Am. J. Hum. Genet., 87, 316-324.

9. de Ligt, J., Willemsen, M.H., van Bon, B.W., Kleefstra, T., Yntema, H.G., Kroes, T., Vulto-van Silfhout, A.T., Koolen, D.A., de Vries, P., Gilissen, C. et al. (2012) Diagnostic exome sequencing in persons with severe intellectual disability. N. Engl. J. Med., 367, 1921-1929.

10. O’Roak, B.J., Deriziotis, P., Lee, C., Vives, L., Schwartz, J.J., Girirajan, S., Karakoc, E., Mackenzie, A.P., Ng, S.B., Baker, C. et al. (2011) Exome sequencing in sporadic autism spectrum disorders identifies severe de novo mutations. Nat. Genet., 43, 585-589.
11. Rauch, A., Wieczorek, D., Graf, E., Wieland, T., Endele, S., Schwarzmayr, T., Albrecht, B., Bartholdi, D., Beygo, J., Di Donato, N. et al. (2012) Range of genetic mutations associated with severe non-syndromic sporadic intellectual disability: an exome sequencing study. Lancet, 380, 1674-1682.

12. Kryukov, G.V., Pennacchio, L.A. and Sunyaev, S.R. (2007) Most rare missense alleles are deleterious in humans: implications for complex disease and association studies. Am. J. Hum. Genet., 80, 727-739.

13. Rousseau, F., el Ghouzzi, V., Delezoide, A.L., Legeai-Mallet, L., Le Merrer, M., Munnich, A. and Bonaventure, J. (1996) Missense FGFR3 mutations create cysteine residues in thanatophoric dwarfism type I (TD1). Hum. Mol. Genet., 5, 509-512.

14. Potocki, L., Abuelo, D.N. and Oyer, C.E. (1995) Cardiac malformation in two infants with hypochondrogenesis. Am. J. Med. Genet., 59, 295-299.

15. Zankl, A., Zabel, B., Hilbert, K., Wildhardt, G., Cuenot, S., Xavier, B., Ha-Vinh, R., Bonafe, L., Spranger, J. and Superti-Furga, A. (2004) Spondyloperipheral dysplasia is caused by truncating mutations in the C-propeptide of COL2A1. Am. J. Med. Genet. A, 129A, 144-148.

16. Nishimura, G., Haga, N., Kitoh, H., Tanaka, Y., Sonoda, T., Kitamura, M., Shirahama, S., Itoh, T., Nakashima, E., Ohashi, H. et al. (2005) The phenotypic spectrum of COL2A1 mutations. Hum. Mutat., 26, 36-43.

17. De Luca, A., Bottillo, I., Sarkozy, A., Carta, C., Neri, C., Bellacchio, E., Schirinzi, A., Conti, E., Zampino, G., Battaglia, A. et al. (2005) NF1 gene mutations represent the major molecular event underlying neurofibromatosis-Noonan syndrome. Am. J. Hum. Genet., 77, 1092-1101.

18. Padmanabhan, A., Lee, J.S., Ismat, F.A., Lu, M.M., Lawson, N.D., Kanki, J.P., Look, A.T. and Epstein, J.A. (2009) Cardiac and vascular functions of the zebrafish orthologues of the type I neurofibromatosis gene NFI. Proc. Natl. Acad. Sci. USA, 106, 22305-22310.

19. Fahsold, R., Hoffmeyer, S., Mischung, C., Gille, C., Ehlers, C., Kucukceylan, N., Abdel-Nour, M., Gewies, A., Peters, H., Kaufmann, D. et al. (2000) Minor lesion mutational spectrum of the entire NF1 gene does not explain its high mutability but points to a functional domain upstream of the GAP-related domain. Am. J. Hum. Genet., 66, 790-818.

20. Boerkoel, C.F., Takashima, H., John, J., Yan, J., Stankiewicz, P., Rosenbarker, L., Andre, J.L., Bogdanovic, R., Burguet, A., Cockfield, S. et al. (2002) Mutant chromatin remodeling protein SMARCAL1 causes Schimke immuno-osseous dysplasia. Nat. Genet., 30, 215-220.

21. Zaidi, S., Choi, M., Wakimoto, H., Ma, L., Jiang, J., Overton, J.D., Romano-Adesman, A., Bjornson, R.D., Breitbart, R.E., Brown, K.K. et al. (2013) De novo mutations in histone-modifying genes in congenital heart disease. Nature, 498, 220-223.

22. Gao, L., Macara, I.G. and Joberty, G. (2002) Multiple splice variants of Par3 and of a novel related gene, Par3L, produce proteins with different binding properties. Gene, 294, 99-107.

23. Hong, E., Jayachandran, P. and Brewster, R. (2010) The polarity protein Pard 3 is required for centrosome positioning during neurulation. Dev. Biol., 341, 335-345.

24. Hirose, T., Karasawa, M., Sugitani, Y., Fujisawa, M., Akimoto, K., Ohno, S. and Noda, T. (2006) PAR3 is essential for cyst-mediated epicardial development by establishing apical cortical domains. Development, 133, 1389-1398.

25. Endele, S., Rosenberger, G., Geider, K., Popp, B., Tamer, C., Stefanova, I., Milh, M., Kortum, F., Fritsch, A., Pientka, F.K. et al. (2010) Mutations in GRIN2A and GRIN2B encoding regulatory subunits of NMDA receptors cause variable neurodevelopmental phenotypes. Nat. Genet., 42, 10211026.

26. Woodbine, L., Neal, J.A., Sasi, N.K., Shimada, M., Deem, K., Coleman, H., Dobyns, W.B., Ogi, T., Meek, K., Davies, E.G. et al. (2013) PRKDC mutations in a SCID patient with profound neurological abnormalities. J. Clin. Invest., 123, 2969-2980.

27. Durkin, M.E., Avner, M.R., Huh, C.G., Yuan, B.Z., Thorgeirsson, S.S. and Popescu, N.C. (2005) DLC-1, a Rho GTPase-activating protein with tumor suppressor function, is essential for embryonic development. FEBS Lett., 579, $1191-1196$.

28. Vilhais-Neto, G.C., Maruhashi, M., Smith, K.T., Vasseur-Cognet, M., Peterson, A.S., Workman, J.L. and Pourquie, O. (2010) Rere controls retinoic acid signalling and somite bilateral symmetry. Nature, $\mathbf{4 6 3}$, 953-957.

29. Plaster, N., Sonntag, C., Schilling, T.F. and Hammerschmidt, M. (2007) REREa/Atrophin-2 interacts with histone deacetylase and Fgf8 signaling to regulate multiple processes of zebrafish development. Dev. Dyn., 236, $1891-1904$. 
30. Kim, B.J., Zaveri, H.P., Shchelochkov, O.A., Yu, Z., Hernandez-Garcia, A., Seymour, M.L., Oghalai, J.S., Pereira, F.A., Stockton, D.W., Justice, M.J. et al.. (2013) An allelic series of mice reveals a role for RERE in the development of multiple organs affected in chromosome $1 \mathrm{p} 36$ deletions. PLoS One, 8, e57460.

31. Goryunov, D., He, C.Z., Lin, C.S., Leung, C.L. and Liem, R.K. (2010) Nervous-tissue-specific elimination of microtubule-actin crosslinking factor 1a results in multiple developmental defects in the mouse brain. Mol Cell. Neurosci., 44, 1-14.

32. Pitera, J.E., Turmaine, M., Woolf, A.S. and Scambler, P.J. (2012) Generation of mice with a conditional null Fraser syndrome 1 (Fras1) allele. Genesis, $\mathbf{5 0}$ 892-898.

33. Vrontou, S., Petrou, P., Meyer, B.I., Galanopoulos, V.K., Imai, K., Yanagi, M., Chowdhury, K., Scambler, P.J. and Chalepakis, G. (2003) Fras1 deficiency results in cryptophthalmos, renal agenesis and blebbed phenotype in mice. Nat. Genet., 34, 209-214.

34. Talbot, J.C., Walker, M.B., Carney, T.J., Huycke, T.R., Yan, Y.L., BreMiller, R.A., Gai, L., Delaurier, A., Postlethwait, J.H., Hammerschmidt, M. et al. (2012) fras 1 shapes endodermal pouch 1 and stabilizes zebrafish pharyngeal skeletal development. Development, 139, 2804-2813.

35. Bisschoff, I.J., Zeschnigk, C., Horn, D., Wellek, B., Riess, A., Wessels, M., Willems, P., Jensen, P., Busche, A., Bekkebraten, J. et al. (2013) Novel mutations including deletions of the entire OFD1 gene in 30 families with type 1 orofaciodigital syndrome: a study of the extensive clinical variability. Hum. Mutat., 34, 237-247.

36. Berger, I., Ben-Neriah, Z., Dor-Wolman, T., Shaag, A., Saada, A., Zenvirt, S., Raas-Rothschild, A., Nadjari, M., Kaestner, K.H. and Elpeleg, O. (2011) Early prenatal ventriculomegaly due to an AIFM1 mutation identified by linkage analysis and whole exome sequencing. Mol. Genet. Metab., 104, $517-520$.

37. Ravenscroft, G., Miyatake, S., Lehtokari, V.L., Todd, E.J., Vornanen, P., Yau, K.S., Hayashi, Y.K., Miyake, N., Tsurusaki, Y., Doi, H. et al. (2013) Mutations in KLHL40 are a frequent cause of severe autosomal-recessive nemaline myopathy. Am. J. Hum. Genet., 93, 6-18.

38. Mefford, H.C., Clauin, S., Sharp, A.J., Moller, R.S., Ullmann, R., Kapur, R., Pinkel, D., Cooper, G.M., Ventura, M., Ropers, H.H. et al. (2007) Recurrent reciprocal genomic rearrangements of $17 \mathrm{q} 12$ are associated with renal disease, diabetes, and epilepsy. Am. J. Hum. Genet., 81, $1057-1069$.

39. Robinson, P.N. and Mundlos, S. (2010) The human phenotype ontology. Clin. Genet., 77, 525-534.

40. Ramu, A., Noordam, M.J., Schwartz, R.S., Wuster, A., Hurles, M.E., Cartwright, R.A. and Conrad, D.F. (2013) DeNovoGear: de novo indel and point mutation discovery and phasing. Nat. Methods, $\mathbf{1 0}$ 985-987. 